\title{
Biokinetic analyses of the latest actinide model for internal dose assessment after an accidental inhalation at a nuclear facility in Oarai, Japan
}

\author{
Kotaro Tani ${ }^{1, *}$, Nobuhito Ishigure ${ }^{1}$, Eunjoo $\mathrm{Kim}^{1}$, Osamu Kurihara $^{1}$ \\ ${ }^{1}$ National Institutes for Quantum and Radiological Science and Technology (QST), National Institute \\ of Radiological Sciences (NIRS), 4-9-1 Anagawa, Inage-ku 263-8555 Chiba, Japan
}

\section{Introduction}

An accidental inhalation of actinide nuclides, e.g. plutonium and americium, by 5 workers occurred due to an accident at Oarai Research and Development Institute, Japan Atomic Energy Agency in June 2017. Although wearing half-face masks, overalls and gloves, the workers possibly inhaled the radionuclides through tiny gaps between their faces and masks. In vivo counting by using a lung monitor and bioassay measurements for urinal and faecal samples have been performed at the National Institute of Radiological Sciences (NIRS) to assess their intake amounts and internal doses. Decorporation agents, i.e. Caand/or Zn-DTPA, were intravenously administered to the all workers for the purpose of enhancing the urinal excretion of actinide nuclides in blood, leading to difficulty in internal dose assessment because the biokinetics may deviate from the general model prediction. The effective dose of the worker who received the highest exposure is currently estimated, still as an initial assessment, to be less than $200 \mathrm{mSv}$ based on previous biokinetic models recommended by International Commission on Radiological Protection (ICRP) [1-3]. The modified biokinetic models for plutonium [4,5], however, had been proposed to reflect data become newly available since development of the previous models. Recently, ICRP also has published some relevant recommendations describing revised biokinetic models for several elements as the Occupational Intakes of Radionuclides (OIR) series [6-8]. In this study, as part of our effort on improvement of the internal dose assessment for the workers, biokinetic characteristics after inhalation of Pu-239 and Am-241 based on the latest models are compared with those based on the previous models.

\section{Methods}

Simultaneous differential equations written according to the previous/latest models shown in Table 1 were numerically analysed by using a commercial software, EQUATRAN-G (Omega Simulation, Japan). Absorption type of the respiratory models was selected to be Type M, and particle size in aerosol was selected to be $1,5,10,15$ or $20 \mu \mathrm{m}$ in activity median aerodynamic diameter (AMAD). Relationships between AMAD and activity ratio, [(Excreted activity in faeces collected over first 5 days)/(Residual activity in the lung (the

* Corresponding author: tani.kotaro@qst.go.jp 
thoracic airways and the thoracic lymph nodes) at third day after intake)], were compared between the previous and latest models.

Table 1. Previous and latest biokinetic models used for comparison.

\begin{tabular}{ccc}
\hline Type of Biokinetic model & Previous model & Latest model \\
\hline Respiratory tract model & ICRP Publ. 66 [2] & ICRP Publ. 130 (OIR part 1) [6] \\
Alimentary tract model & ICRP Publ. 30 [1] & ICRP Publ. 100 [9] \\
Systemic model & ICRP Publ. 67 [3] & Leggett et al. [5] \\
\hline
\end{tabular}

\section{Results}

Relationships between AMAD and the activity ratio are shown in Figure 1 (a) Pu-239 and (b) Am-241, resulting in large differences between the previous and latest models. This activity ratio is one of important facts, obtained from results of in vivo counting and bioassay measurements, to estimate $\mathrm{AMAD}$, especially as in this case that information on particle size in aerosol is unavailable. The actual activity ratio of Am-241 measured after the accident was about 34, corresponding to more than $17 \mu \mathrm{m}$ and $12 \mu \mathrm{m}$ based on the previous and latest models. The results of this study demonstrate that AMAD is overestimated based on the previous models, leading to underestimation of actinide amount absorbed from respiratory tracts into blood because smaller particle in aerosol is likely to be inhaled deeply into the lungs. Therefore, it is clearly worth considering to apply the latest models in future studies for improvement of the internal dose assessment for the workers.

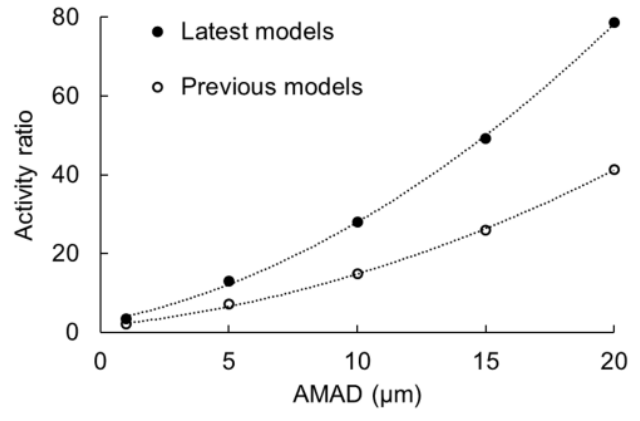

(a) $\mathrm{Pu}-239$

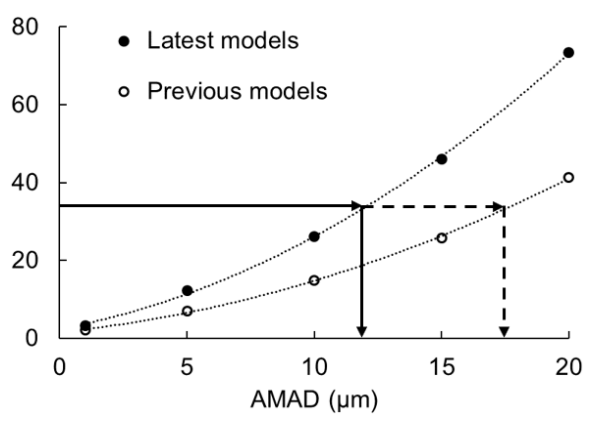

(b) Am-241

Figure 1. Relationships between AMAD and activity ratio: [(Faecal activity for 5 days)/(Lung activity at third day)]. 


\section{References}

1. ICRP Publication 30 (Part 1), Ann. ICRP 2, 3-4 (1979) ; 2. ICRP Publication 66, Ann. ICRP 24, 1-3 (1994) ; 3. ICRP Publication 67, Ann. ICRP 23, 3-4 (1993) ; 4. Leggett, R.W., Radiat. Prot. Dosim. 106, 103-120 (2003) ; 5. Leggett, R.W. et al, Radiat. Res. 164, 111122 (2005) ; 6. ICRP Publication 130, Ann. ICRP 44, 2 (2015) ; 7. ICRP Publication 134, Ann. ICRP 45, 3-4 (2016) ; 8. ICRP Publication 137, Ann. ICRP 46, 3-4 (2017) ; 9. ICRP Publication 100, Ann. ICRP 36, 1-2 (2006). 